\title{
Constitution d'un réseau pour la gestion des ressources génétiques des céréales à paille
}

\author{
A Le Blanc, J Koenig \\ Groupement d'étude et de contrôle des variétés et des semences, \\ et Institut national de la recherche agronomique, Domaine de Crouëlle, \\ Station d'amélioration des plantes, F63039 Clermont-Ferrand Cedex 02, France
}

Résumé - La gestion des ressources génétiques des céréales à paille en France est réalisée au travers d'un réseau coopératif assisté d'un comité de pilotage et associant des partenaires publics et privés, les conservateurs, et une unité coordinatrice. Les partenaires ainsi que leurs rôles respectifs sont définis par une une charte et un règlement intérieur. Le financement du programme est régi par une convention triennale associant des financeurs directs : le ministère de l'Agriculture, l'ONIC (Office national interprofessionnel des céréales) et le BRG (Bureau des ressources génétiques) et des financeurs indirects : l'INRA (Institut national de la recherche agronomique) et le SPSS (Syndicat des producteurs de semences sélectionnées); le GEVES (Groupement d'étude et de contrôle des variétés et des semences) est gestionnaire du budget et employeur du coordinateur. L'objectif de ce réseau est d'assurer la sauvegarde et la valorisation d'une collection nationale dont le contenu dépend des objectifs fixés par les partenaires, de recommandations européennes et des connaissances actuelles sur la diversité.

ressource génétique / céréale à paille / réseau / conservation / évaluation

Summary - Setting up a network for management of small grain genetic resources. In France, small grain genetic resources are managed by a cooperative network which associates public and private partners, the curators, and a coordination unit. This network is controlled by a steering committee. The partners, their roles and the working of the network are defined in a charter with internal regulations. Funding is supported by the Ministry of Agriculture, the ONIC and the BRG, under a 3-year agreement; INRA and SPSS fund the programme indirectly and GEVES manages the finances and employs the coordinator. The aim of this network is to ensure the safeguard and the exploitation of a national small grain collection; the partners define the introduction criteria according to their own objectives, European recommendations and current knowledge about diversity.

genetic resources / small grain / network / conservation / evaluation 


\section{INTRODUCTION}

Depuis les années 1964-65, un certain nombre d'initiatives ont permis de véhiculer et populariser la notion de sauvegarde de la variabilité génétique; il faut citer notamment en ce qui concerne les céréales à paille, le travail de sensibilisation et de proposition mené par André Cauderon, d'abord au sein d'une mission semences puis par le canal du Bureau des ressources génétiques qu'il dirigeait. Des actions concrètes ont alors été lancées : informatisation de collections, éditions de listes plus ou moins renseignées, diffusion de catalogues...

Ainsi, sous l'impulsion de Cauderon (1985) et suite à sa proposition de créer un centre de ressources génétiques pour les céréales, une action concertée a réuni à partir de 1987 : le ministère de l'Agriculture, l'ONIC (Office national interprofessionnel des céréales), l'INRA, le BRG (Bureau des ressources génétiques), l'ITCF (Institut technique des céréales et fourrages), le SPSS (Syndicat des producteurs de semences sélectionnées) et le GNIS (Groupement national interprofessionnel des semences).

L'inventaire des ressources génétiques des céréales (blé et orge en premier lieu puis avoine, triticale et seigle) disponibles en France constituait le principal objectif de l'opération; l'intérêt qu'il a suscité chez tous les détenteurs, une trentaine au total dans le secteur privé, plus les différents laboratoires céréales INRA, a conduit son comité de pilotage à s'engager dans la constitution d'une unité coordinatrice pour les céréales à paille. Ce nouvel objectif rassemble les mêmes partenaires, plus le GEVES qui, en recrutant le coordonnateur et animateur du programme, se présente comme un élément moteur, avec l'INRA, en matière de préservation et de gestion de la diversité génétique.

\section{QUI CONSERVE DES RESSOURCES GÉNÉTIQUES DE CÉRÉALES EN FRANCE?}

Le GEVES maintient des collections importantes de céréales liées à sa mission d'examen des variétés présentées en vue de leur inscription au catalogue des espèces et variétés de plantes cultivées.

L'INRA introduit annuellement un certain nombre de génotypes issus d'échanges avec des instituts étrangers ou de pépinières internationales et sur lesquels des évaluations sont faites avant une éventuelle utilisation en tant que géniteurs, un transfert en collection et/ou en réseau coopératif d'observation des ressources génétiques.

Cinq laboratoires (Clermont-Ferrand, Dijon, Montpellier, Rennes et VersaillesMons) détiennent des collections de blé tendre. Celles-ci représentent plus de 8000 accessions pour environ 5700 génotypes différents. Une collection de blés durs représentant quelque 1000 accessions est maintenue à Montpellier. Une grande partie de la collection ORGE-INRA (environ 3500 génotypes) est hébergée à Clermont-Ferrand. Une série de génotypes résistants à la sécheresse est maintenue à Montpellier. L'informatisation de la collection Triticale, à Clermont-Ferrand est en cours et concerne environ 300 génotypes. Peu d'efforts de maintien et de codage ont été entrepris sur le seigle et l'avoine qui ne font plus l'objet de programmes de 
recherche à l'INRA. Mais les inventaires sont en cours de manière à organiser la sauvegarde de ce patrimoine.

L'implication des organismes privés dans le maintien des collections est variable selon les sociétés et a évolué au cours du temps. Bien souvent, l'objectif des collections privées est de fournir une variabilité génétique directement valorisable dans les programmes de sélection en cours. De ce fait, les collections sont dans la plupart des cas restreintes ou du moins peu ouvertes à des échanges. Certains organismes cependant continuent à maintenir des collections importantes (SECOBRA en orge, par exemple) ; 1100 génotypes de blé et 300 d'orge, candidats potentiels à la collection nationale car différents des génotypes maintenus dans les collections publiques ont été inventoriés.

Des collections de céréales à paille existent dans d'autres organismes tels que des lycées agricoles, l'ITCF, des conservatoires ou jardins botaniques. Ces collections ont le plus souvent un objectif pédagogique ou de démonstration en direction du public, notamment en ce qui concerne les blés anciens français et présentent des génotypes qui sont pour la plupart déjà répertoriés à l'INRA.

\section{LE RÉSEAU COOPÉRATIF DE GESTION DES RESSOURCES GÉNÉTIQUES DES CÉRÉALES À PAILLE}

Le premier objectif de ce réseau est de gérer une collection nationale identifiée, répertoriée, localisée et disponible dont l'accès est libre et gratuit dans des limites raisonnables décrites dans un règlement intérieur; cette disponibilité est basée sur un principe d'échanges et de réciprocité.

Un deuxième objectif est d'évaluer tous les ans dans les différentes implantations du réseau une série de génotypes de blés tendres, orges d'hiver et de printemps pour des caractères utiles au sélectionneur : résistance aux principaux parasites, précocité. Des caractères complémentaires (valeur d'utilisation, diagrammes d'électrophorèses des protéines de réserve du grain) sont évalués sur le grain récolté.

La gestion des ressources génétiques est réalisée par l'ensemble des partenaires, les conservateurs, assistés d'une unité coordinatrice. Le fonctionnement du réseau est placé sous le contrôle d'un comité de pilotage.

Les partenaires et le mode de fonctionnement du réseau sont définis dans une charte et un règlement intérieur.

\section{L'unité coordinatrice}

Elle est basée à la station d'amélioration des plantes du centre INRA de ClermontFerrand et animée par un ingénieur du GEVES. Les rôles qui lui ont été confiés sont : l'animation du réseau coopératif, l'enrichissement et la gestion de la base de données, l'édition et la diffusion de catalogues, des recherches sur la méthodologie de gestion de la variabilité et sur l'estimation de la diversité, la représentation européenne et internationale. Plus précisément, la tâche d'animation du réseau consiste à : organiser et coordonner les réseaux d'évaluation par espèce et de maintien de la collection nationale, organiser la répartition de la collection nationale 
chez les différents partenaires, enrichir la collection nationale, suivre les préévaluations réalisées par l'INRA, gérer le double de sécurité de la collection nationale, centraliser les demandes de semences et distribuer les échantillons correspondants.

\section{Les conservateurs}

Il s'agit de stations du DGAP (Département de génétique et d'amélioration des plantes) de l'INRA impliquées dans l'amélioration génétique des céréales, les établissements de sélection, membres ou non du SPSS, le GEVES-Station du Magneraud - et d'une manière générale toute structure, publique ou privée acceptée par le comité de pilotage et adhérant à la présente charte. Ils ont pour rôles : la participation au pilotage du programme et à la constitution de la collection nationale, la collecte et les préévaluations de nouveaux génotypes, le maintien de l'entité génétique et la conservation du pouvoir germinatif des génotypes de la collection nationale en fonction de la répartition établie en accord avec les conservateurs, la distribution des semences (conservateurs INRA uniquement) et les évaluations dans le cadre des réseaux d'observation des ressources génétiques.

Les rôles respectifs des différents partenaires peuvent être résumés dans le tableau synoptique (tableau I).

Tableau I. Répartition des tâches de gestion des ressources génétiques des céréales à paille entre les conservateurs et l'unité coordinatrice du réseau.

\begin{tabular}{|c|c|c|}
\hline Les tâches & Les acteurs & \\
\hline $\begin{array}{l}\text { Introduction de } \\
\text { nouveaux génotypes }\end{array}$ & $\begin{array}{l}\text { Les conservateurs } \\
\text { Proposent }\end{array}$ & $\begin{array}{l}\text { Unité coordinatrice } \\
\text { Accepte et enregistre } \\
\text { les nouvelles introductions }\end{array}$ \\
\hline Maintien & Sont responsables du maintien & \\
\hline Stockage & $\begin{array}{l}\text { Assurent le stockage : } \\
\text { - des semences pour le renouvellement } \\
\text { - des épis de référence }\end{array}$ & $\begin{array}{l}\text { Assure le stockage } \\
\text { - du double de sécurité } \\
\text { - des semences pour la } \\
\text { distribution }\end{array}$ \\
\hline Distribution & $\begin{array}{l}\text { S'ils sont INRA, distribuent les } \\
\text { semences qui sont sous leur } \\
\text { responsabilité }\end{array}$ & $\begin{array}{l}\text { Centralise les demandes et } \\
\text { distribue les semences qui } \\
\text { sont sous sa responsabilité }\end{array}$ \\
\hline Base de données & & En assure la gestion \\
\hline Évaluation & Observent & Réalise la synthèse \\
\hline Coordination & $\begin{array}{l}\text { Participent au } \\
\text { Comité de pilotage }\end{array}$ & $\begin{array}{l}\text { Coordonne le réseau sous } \\
\text { la responsabilité d'un } \\
\text { comité de pilotage }\end{array}$ \\
\hline
\end{tabular}




\section{LE COMITÉ DE PILOTAGE}

Il rassemble un représentant du ministère de l'Agriculture, le responsable de l'unité coordinatrice, un représentant du GEVES, un représentant de l'ONIC, un représentant du BRG, un représentant du DGAP de l'INRA, un représentant du SPSS, le secrétaire technique de la section céréales à paille du CTPS, un représentant du GNIS, 6 représentants d'établissements membres du SPSS et du réseau et 6 représentants de stations du DGAP de l'INRA impliquées dans le réseau. Il peut en outre faire appel, de manière ponctuelle, à des experts.

Il est présidé par le représentant du ministère de l'Agriculture et animé par le responsable de l'unité coordinatrice, coordinateur du réseau.

Il se réunit au moins une fois par an pour : déterminer la politique générale du réseau, fixer par un règlement intérieur les modalités de gestion de la collection nationale (introduction, retrait, maintien, conservation...) et d'accès aux ressources génétiques nationales, faire des propositions concernant les programmes de l'unité coordinatrice, l'organisation du réseau et son évolution juridique, faire le point sur les actions engagées par les partenaires et l'unité coordinatrice dans le cadre du réseau, examiner son ouverture à de nouveaux partenaires français ou étrangers, rechercher les moyens nécessaires pour soutenir son fonctionnement.

\section{CONSTITUTION DE LA COLLECTION NATIONALE}

Face à la décision d'entrée en collection nationale, il est nécessaire de «définir les voies les plus simples pour réaliser un choix représentatif de la diversité génétique d'une espèce ou d'un groupe d'espèces» (Doussinault, 1992, Les ressources génétiques au DGAP - présentation faite au conseil scientifique du département le 7 novembre 1991).

Les partenaires de programme ont arrêté un certain nombre de critères leur permettant de choisir dans leurs collections respectives les génotypes à conserver en priorité dans le cadre d'une collection nationale. Ces critères (d'introduction ou de retrait) pourront évoluer en fonction des recherches entreprises par l'unité coordinatrice sur la constitution de core collections et sur l'estimation de la diversité. En tout état de cause, une variété qui serait maintenue en collection de référence à des fins d'inscription à un catalogue ou de protection des obtentions végétales ne peut être proposée en collection nationale.

\section{Critères d'introduction en collection nationale}

Les différents critères sont énoncés ci-dessous :

- a) les cultivars français radiés du catalogue français des variétés (listes $\mathrm{A}$ et B) : dès lors qu'une variété est radiée du catalogue français, elle rentre en collection nationale et peut être distribuée en tant que ressource génétique;

- b) les populations et variétés de pays françaises : ce point représente un nombre limité et peu extensible de ressources génétiques. Tout ce qui est actuellement décrit dans les différentes collections peut être enregistré en collection nationale, sans restriction sur les éventuelles redondances ou ressemblances. En ce qui concerne le maintien en isolement des populations retenues pour la collection nationale, il 
pourrait être assuré par des conservatoires, jardins botaniques, musées... de leurs régions d'origine, à l'exemple des échanges mis en place entre la station de Rennes (Le Rheu) et l'écomusée de la Bintinais de la ville de Rennes;

- c) les géniteurs français ou étrangers : il s'agit notamment de ceux ayant donné dans leur descendance des variétés inscrites au catalogue français ou européen, la décision de proposer ce matériel pour la collection nationale revenant à l'obtenteur (ou le détenteur, si le géniteur n'est pas confidentiel) qui connaît l'intérêt de ce matériel et sa disponibilité;

- d) le matériel original issu de prospection en zone de diversification;

- e) le matériel connu pour la présence de gènes identifiés, comme les génotypes servant de témoins dans certaines pépinières ou essais, les hôtes différentiels...;

- f) le matériel peu connu mais reconnu comme ressource génétique et dont la fourniture ou la collecte reste difficile;

Il a été proposé de ne pas introduire en collection nationale le matériel étranger connu, classé et publié par d'autres banques de gènes et ne répondant pas aux critères $\mathrm{c}, \mathrm{d}$ et e, à condition que la fourniture de semences de bonne qualité soit possible, de manière aisée et dans des délais raisonnables.

En attendant de disposer de critères plus efficaces d'appréhension de la variabilité, le comité de pilotage du programme recommande la prudence dans l'application de ces critères d'introduction, pour limiter à la fois la redondance et la perte de matériel et ne pas atteindre des effectifs qui ne seraient plus correctement gérables.

\section{Critères de retrait de la collection nationale}

Là encore, la prudence est recommandée car les motifs d'élimination ne tiennent pas compte d'intérêts ultérieurs éventuels, inconnus au moment du retrait. Les cas de retrait sont :

- a) redondance évidente, partielle ou totale;

- b) présence d'un gène connu dans du matériel plus adapté.

Le retrait de la collection nationale n'implique pas le retrait de la collection d'origine.

\section{CONCLUSION}

L'opération «Inventaire des ressources génétiques des céréales à paille» a marqué le début d'une action systématique réunissant en réseau des partenaires publics et privés, pour organiser la sauvegarde, l'étude et la valorisation du potentiel français en matière de ressources génétiques de céréales.

Outre l'enrichissement des ressources génétiques disponibles pour la sélection, cet inventaire a permis de créer une dynamique d'échanges entre les secteurs privé et public en matière de ressources génétiques, et initié un partenariat original dans le domaine de la gestion de ces ressources. L'évaluation en réseau de ressources génétiques utilisables par la sélection comme géniteurs est désormais bien établie pour le blé et l'orge; certains caractères évalués comme la tolérance aux mosaïques du blé et de l'orge présentent un grand intérêt pour les sélectionneurs. La collection nationale, qui devrait intégrer ces génotypes et tous ceux des collections 
individuelles répondant aux critères définis par les partenaires du programme, devrait voir le jour en 1994. Cette collection constituera une première étape dans la constitution d'une core collection : Frankel et Brown (1984), Knüpfer (1992) ont déjà donné leur point de vue sur le sujet. Une base de données déjà structurée est en cours de chargement avec l'ensemble des informations recueillies jusqu'à maintenant dans les collections individuelles et dans les réseaux d'évaluations.

\section{REMERCIEMENTS}

Les auteurs remercient les divers partenaires cités dans le présent article et tout particulièrement le ministère de l'Agriculture et de la Pêche, l'ONIC et le BRG qui financent directement le programme national de gestion coopérative des ressources génétiques des céréales à paille.

\section{RÉFÉRENCES}

Cauderon A (1985) Un projet de centre français de ressources génétiques pour les céréales. Perspec Agric 98, 58-63

Frankel OH, Brown AHD (1984) Plant genetic resources today: a critical appraisal. In: Crop genetic resources: conservation and evaluation (Holden W, George Allen and Unwin London, eds). UK, 249-257

Knüpfer H (1992) The barley core collection. An international effort. In: Proc Int IBPGR/CENARGEN workshop on core collection, Brazilia 